\title{
ORIF versus arthroplasty for open proximal humerus fractures: Nationwide Inpatient Sample data between 1998 and 2013
}

Anant Dixit, Frank S. Cautela, Colin S. Cooper, George A. Beyer, James C. Messina, Jeffrey E. Mait, Neil V. Shah, Bassel G. Diebo*, Carl B. Paulino and William P. Urban

\begin{abstract}
Background: Limited data exists in analyzing open reduction and internal fixation (ORIF) and arthroplasty in the management of open proximal humerus fractures. We analyzed differences in hospital course between these procedures, patient demographics, complication rate, length of stay, hospital charges, and mortality rate.

Materials and methods: This is a retrospective review of the Nationwide Inpatient Sample database. ICD-9 codes identified patients hospitalized for open proximal humerus fractures from 1998 to 2013 who underwent ORIF or shoulder arthroplasty (hemi-, total, or reverse). Demographics and in-hospital complications were compared. Logistic regression controlling for age, gender, and Deyo index tested the impact of ORIF Vs ARTH on any complications.

Results: Seven hundred thirty patients were included ( $\mathrm{ORIF}, n=662$ vs ARTH, $n=68$ ). ORIF patients were younger $(p<0.001)$, more likely to be males $(p<0.001)$, and had a lower Deyo score $(p=0.012)$. Both groups had comparable complication rates ( $21.4 \%$ vs $18.0 \%, p=0.535$ ), lengths of stay ( 7.86 days vs 7.44 days, $p=0.833$ ), hospital charges $(\$ 76,998$ vs $\$ 64,133, p=0.360)$, and mortality rates $(0.2 \%$ vs $0 \%, p=0.761)$. Type of surgery was not a predictor of any complications $(\mathrm{OR}=0.67$ [95\% $\mathrm{Cl} 0.33-1.35], p=0.266)$, extended length of stay $(\mathrm{OR}=1.01$ [95\% $\mathrm{Cl} 0.58-1.78]$, $p=0.967)$, or high hospital charges ( $\mathrm{OR}=1.39[95 \% \mathrm{Cl} 0.68-2.86], p=0.366)$.
\end{abstract}

Conclusion: We revealed no differences in hospital course between ORIF and arthroplasty for management of open proximal humerus fractures. Although differences in demographics existed, no differences in complication rates, length of stay, hospital charges and mortality rates were noted. Future studies can evaluate the long-term outcomes of these procedures.

Level of evidence: Level III.

Keywords: ORIF, Arthroplasty, Shoulder, Proximal humerus, Open fracture, Nationwide Inpatient Sample (NIS)

\section{Introduction}

Proximal humerus fractures are the third most common fracture in adults, representing almost $6 \%$ of all adult fractures and half of fractures of the humerus [1-3]. They are typically associated with minimal trauma in the elderly, particularly in osteoporotic individuals, and with

\footnotetext{
*Correspondence: Dr.basseldiebo@gmail.com;

Bassel.Diebo@Downstate.edu

Department of Orthopaedic Surgery and Rehabilitation Medicine, State University of New York, Downstate Medical Center, 450 Clarkson Avenue, Box 30, Brooklyn, NY 11203, USA
}

high-energy trauma in younger patients. Most of these fractures can be treated conservatively with satisfactory results [4]. More complex fractures may require surgical intervention, such as with open reduction and internal fixation (ORIF) or arthroplasty (either total, reverse total, or hemiarthroplasty). Currently, there is conflicting data in the literature with regards to which surgical technique is most effective at treating fractures of the proximal humerus. Even though it appears as if ORIF is the technique preferred for younger patients, there is no consensus on which treatment option is most preferred or effective for all patients [5]. While it has been shown 
to demonstrate better functional outcomes and restoration of anatomy, ORIF can also be associated with higher reoperation rates $[6,7]$.

Open proximal humerus fractures in particular are a relatively rare but serious injury. One study found that out of 1582 proximal humerus fractures over a 2-year period, only $3(0.2 \%)$ were open [8]. Open humeral fractures in general tend to be associated with high energy trauma, such as motor vehicle accidents $[8,9]$. These injuries can be associated with more serious sequelae, such as injury to the axillary artery [10]. Due to the fact that they are relatively uncommon, there is a paucity of literature analyzing specifically open proximal humerus fractures and management.

Due to the conflicting nature of the literature, lack of data, and the importance of the topic, we utilized the Nationwide Inpatient Sample (NIS) database to analyze the differences in the hospital course of patients who were treated with ORIF vs arthroplasty for open fractures of the proximal humerus. Specifically, we sought to do this in terms of demographics, complication rate, length of stay, hospital charges, and mortality rate.

\section{Materials and methods}

\section{Data source}

All data was used from a subset generated from the Nationwide Inpatient Sample (NIS) spanning the years 1998-2013. NIS is supported by the Health Care Cost and Utilization Project (HCUP) and sponsored through both federal, state, and industry partnerships. The NIS database provides the most robust, publicly available data for inpatient care across all-payers in the United States. In its entirety, more than seven million individual hospital visits per year were recorded within the data set. Our institutional review board approved this study as exempt due to the de-identified nature of the data. Inclusion criteria were patients admitted with ICD-9 codes of primary diagnoses of open proximal humerus fractures (812.10, $812.11,812.12,812.13,812.19)$. Patients with multitrauma or pathologic fractures were excluded.

\section{Data collection}

The demographics studied include age, gender, race, Deyo index, and type of insurance as reported in the NIS dataset. Using ICD-9 procedure codes, patients that underwent either ORIF (79.31) or arthroplasty (reverse 81.88, total 81.80, partial 81.81) following open proximal humerus fractures were grouped into the corresponding subset. In-hospital complications were grouped based on related sets of diagnoses as follows: infection, post-hemorrhagic anemia, cardiac complications, respiratory complications, device-related complications, post-operative shock, hematoma formation, pulmonary insufficiency, deep vein thrombosis (DVT), pulmonary embolism (PE), and any complication. Any complication was defined as the occurrence of at least one complication. Length of hospital stay, total cost of stay, and mortality were also reported.

\section{Study design and statistical analysis}

Descriptive analysis was used to characterize the open proximal humerus fracture population. Patients were stratified based on the surgical repair; ORIF patients comprised the first group, while total, hemi, and reverse arthroplasty patients were combined into a second group. Independent sample $t$-tests were employed to evaluate variation of continuous variables such as age, Deyo index, and total hospital cost between the two groups. Accordingly, chi-square analysis was used to evaluate the potential association between gender, race, insurance type, and surgical procedure. In evaluating complications between patients that underwent ORIF or arthroplasty, Chi-square analysis was conducted to evaluate the potential variation between the surgical groups on the basis of infection, post-hemorrhagic anemia, cardiac complications, respiratory complications, device-related complications, post-operative shock, hematoma formation, pulmonary insufficiency, DVT, any in-hospital complication and mortality.

A logistic regression model controlling for age, gender, Deyo score, and type of procedure was utilized to identify independent predictors of any complication, extended length of stay, and greater hospital charges. Extended length of stay and greater hospital charges were defined as $\geq 60$ th percentile of the overall cohort. The level of significance was set to $p$ value $<0.05$. All analysis was performed using IBM SPSS Statistics 24. There was no potential source of bias during this analysis. In order to investigate the association of age and the complications of interest, increased length of stay, total charges, and the occurrence of any complication, an additional analysis was performed on a subset of the dataset containing only patients older than 45 years of age.

\section{Results}

\section{Overall patient demographics}

Analysis of NIS identified an estimated 1413 patients with open proximal humerus fractures with a mean age of $47.62 \pm 23.2$ years and $59.4 \%$ of patients being males. A plurality $(28.9 \%)$ of patients had private insurance and the majority (50.3\%) were white. The most common causes of fractures were falls, gunshots, motor vehicle accidents, and suicide attempts. Detailed demographics are reported in Table 1.

The most common procedures were: ORIF $(n=662)$, arthroplasty $(n=68)$ and open reduction without internal 
Table 1 Demographics of all patients with proximal humerus fractures

\begin{tabular}{ll}
\hline Patient demographics & $\begin{array}{l}\text { All patients } \\
\text { with proximal } \\
\text { humerus fractures }\end{array}$ \\
\hline Sample size & 1413 \\
Mean age (years) & 47.6 \\
Deyo index & 0.41 \\
Gender & \\
Male & $59.40 \%$ \\
Female & $39.90 \%$ \\
Race & \\
White & $50.30 \%$ \\
Black & $16.60 \%$ \\
Hispanic & $8.80 \%$ \\
Other & $3.50 \%$ \\
Data missing & $20.80 \%$ \\
Insurance & \\
Private insurance & $28.90 \%$ \\
Medicare & $27.40 \%$ \\
Self-pay & $16.10 \%$ \\
Medicaid & $15.10 \%$ \\
No charge & $1.60 \%$ \\
Other & $10.30 \%$ \\
\hline
\end{tabular}

Table 2 Most common procedures for open proximal humerus fractures

\begin{tabular}{llr}
\hline Procedure & $\boldsymbol{n}$ \\
\hline Procedure & ORIF & 662 \\
& Arthroplasty & 68 \\
Shoulder hemiarthroplasty & 59 \\
Reverse shoulder arthroplasty & 5 \\
Total shoulder arthroplasty & 4 \\
Open reduction without internal fixation & 33 \\
Internal fixation without reduction & 28 \\
Closed reduction without internal fixation & 26 \\
External fixation & 22 \\
Amputation through humerus & 9 \\
Other/not coded & 565 \\
Total & 1413
\end{tabular}

fixation $(n=33)$. All available procedures are listed in Table 2.

\section{ORIF vs arthroplasty}

Seven hundred thirty patients who underwent either ORIF $(n=662)$ or arthroplasty $(n=68)$ procedures were further analyzed (Table 3). ORIF patients were younger than arthroplasty patients $(48.6 \pm 21.8$ vs $64.9 \pm 17.7$, $p<0.001)$ with a lower Deyo score $(0.41 \pm 0.87$ vs $0.7 \pm 0.97, p=0.012$ ). In terms of gender, $57.9 \%$ of patients undergoing ORIF vs $30.5 \%$ of patients undergoing arthroplasty were male $(p<0.001)$. Race varied, with the majority being white in both groups; however, $15.3 \%$ of ORIF patients were black vs $0 \%$ of arthroplasty $(p=0.004)$. There was a difference in health insurance coverage between the groups with $33.0 \%$ of ORIF patients being covered by private insurance vs most arthroplasty patients $(55.7 \%)$ covered by Medicare $(p<0.001)$ (Table 3).

\section{Surgical complications and hospital course}

There was no difference in total complication rate between ORIF and arthroplasty $(21.4 \%$ vs $18.0 \%$, $p=0.535)$. Specifically, there was no difference with respect to post-hemorrhagic anemia $(15.4 \%$ vs $13.1 \%$, $p=0.636)$, pulmonary insufficiency $(4.2 \%$ vs $0 \%$, $p=0.102)$, infection $(1.1 \%$ vs $1.6 \%, p=0.677)$ and device-related complications $(0.5 \%$ vs $1.6 \%, p=0.231)$. The length of stay in ORIF patients was $7.86 \pm 14.9$ days while length of stay in arthroplasty patients was $7.44 \pm 12.1$ days $(p=0.833)$. Total charges were comparable, with a mean total cost of $\$ 76,998$ for ORIF patients and $\$ 64,133$ for arthroplasty $(p=0.833)$. Despite the fact that one patient from the ORIF group died, the mortality rate was comparable $(0.2 \%$ vs $0 \%, p=0.761)$ (Table 4$)$. When stratified by age, patients greater than 45 years of age showed the same trends.

Logistic regression revealed that, when using ORIF as a reference, the type of surgery (ORIF vs arthroplasty) was not a predictor of any complications $(\mathrm{OR}=0.67[95 \% \mathrm{CI}$ $0.33-1.35], p=0.266)$, extended length of stay $(\mathrm{OR}=1.01$ [95\% CI 0.58-1.78], $p=0.967$ ), or high hospital charges $(\mathrm{OR}=1.39$ [95\% CI 0.68-2.86], $p=0.366)$. Age was the only independent predictor of sustaining any complication $(\mathrm{OR}=1.01$ [95\% CI 1.003-1.02], $p=0.006)$. Similarly, increased Deyo score was a predictor of extended length of stay $(\mathrm{OR}=1.24$ [95\% CI 1.01-1.51], $p<0.036)$ and greater hospital charges (OR $=1.31$ [95\% CI 1.021.69], $p<0.034)$. Finally, increased age was a predictor of decreased hospital charges $(\mathrm{OR}=0.98$ [0.97-0.99], $p=0.001$ ) (Table 5). Subsequent logistic regression on patients older than 45 revealed the same trends, except for the association between age and the occurrence of any complication, which was not significant within this subset.

\section{Discussion}

This study found that for open proximal humerus fractures, the hospital course of patients undergoing either ORIF or arthroplasty is comparable. There was no statistical difference in the inpatient complication rate 
Table 3 Demographic comparisons of patients with proximal humerus fractures that had ORIF or arthroplasty surgical interventions

\begin{tabular}{|c|c|c|c|}
\hline \multirow[t]{2}{*}{ Patient demographics } & \multicolumn{3}{|c|}{ Surgical procedure } \\
\hline & ORIF & Arthroplasty & $p$ value \\
\hline Sample size & 662 & 68 & - \\
\hline Mean age (years) & 48.6 & 64.9 & $<0.001$ \\
\hline Length of stay (days) & 7.86 & 7.44 & 0.833 \\
\hline Total charges & $\$ 76,998$ & $\$ 64,133$ & 0.360 \\
\hline Deyo index & 0.41 & 0.70 & 0.012 \\
\hline \multicolumn{4}{|l|}{ Gender } \\
\hline Male & $57.9 \%$ & $30.5 \%$ & \multirow[t]{2}{*}{$<0.001$} \\
\hline Female & $42.1 \%$ & $69.5 \%$ & \\
\hline \multicolumn{4}{|l|}{ Race } \\
\hline White & $70.6 \%$ & $86.4 \%$ & \multirow[t]{4}{*}{0.004} \\
\hline Black & $15.3 \%$ & $0 \%$ & \\
\hline Hispanic & $10.5 \%$ & $9.1 \%$ & \\
\hline Other & $3.6 \%$ & $4.5 \%$ & \\
\hline \multicolumn{4}{|l|}{ Insurance } \\
\hline Private insurance & $33.0 \%$ & $19.7 \%$ & \multirow[t]{6}{*}{$<0.001$} \\
\hline Medicare & $28.2 \%$ & $55.7 \%$ & \\
\hline Medicaid & $14.2 \%$ & $1.6 \%$ & \\
\hline Self-pay & $14.0 \%$ & $8.2 \%$ & \\
\hline No charge & $1.4 \%$ & $1.6 \%$ & \\
\hline Other & $9.3 \%$ & $13.1 \%$ & \\
\hline
\end{tabular}

Statistically significant $p$ values are in italics

Table 4 Common surgical complications in ORIF and arthroplasty

\begin{tabular}{lrcl}
\hline Complication & ORIF & Arthroplasty & $\boldsymbol{p}$ value \\
\hline Post-hemorrhagic anemia & $15.4 \%$ & $13.1 \%$ & 0.636 \\
Pulmonary insufficiency & $4.2 \%$ & $0.0 \%$ & 0.102 \\
Infection & $1.1 \%$ & $1.6 \%$ & 0.677 \\
Cardiac complications & $0.9 \%$ & $0.0 \%$ & 0.456 \\
Respiratory complications & $0.9 \%$ & $1.6 \%$ & 0.575 \\
Device-related complications & $0.5 \%$ & $1.6 \%$ & 0.231 \\
Hematoma & $0.5 \%$ & $0.0 \%$ & 0.599 \\
Post-operative shock & $0.3 \%$ & $0.0 \%$ & 0.668 \\
Digestive complications & $0.3 \%$ & $0.0 \%$ & 0.668 \\
Urinary complications & $0.3 \%$ & $0.0 \%$ & 0.668 \\
Deep vein thrombosis & $0.2 \%$ & $0.0 \%$ & 0.761 \\
Pulmonary embolism & $0.0 \%$ & $0.0 \%$ & - \\
Total complication rate & $21.4 \%$ & $18.0 \%$ & 0.535 \\
\hline
\end{tabular}

Note, total complication rate does not represent a sum of all complications, but represents rate of any complication following surgery. The row labels do not represent an inclusive sample of all possible surgical complications

between the two procedures $(21.4 \%$ vs $18.0 \%$, respectively). Age played a minimal role in predicting complications or hospital charges. However, increased underlying comorbidities prior to surgery, as reflected by the Deyo score, was the only predictor of increased length of stay and higher hospital charges in this patient population. Patients who underwent ORIF were younger and more likely to be black, male, and insured by a private insurance company when compared to the arthroplasty cohort.

The findings of this study are comparable to those previously reported in the literature on closed fractures. Thorsness et al. [11] reported no differences in 30-day complication rates and length of hospital stay between hemiarthroplasty and ORIF. This study also reported that patients with proximal humeral fractures undergoing ORIF were younger than patients undergoing hemiarthroplasty. This is most likely due to the quality bone stock available in patients under 65 years old [12]. This may also be due to the desire to preserve more bone in individuals who participate in higher risk activities that may lead to future complications or reoperations. As the results of this study concluded, others also found that ORIF patients were more likely to be male $[11,13]$.

Petrigliano et al. [14] reported that males over 65 years old with preexisting comorbidities had the highest short-term complication rates; this is in line with our findings. However, the mortality rate in our results was comparable between the groups. This is also consistent with Thorsness et al. [11], who found that the mortality rate between the two surgeries was not statistically significant. On the other hand, Neuhaus et al. [15] found a $1.9 \%$ increase in hospital deaths in patients who underwent ORIF compared to arthroplasty. Regarding insurance coverage, Zhang et al. [16] showed that arthroplasty patients had an overall higher Medicare rate, which is similar to the results of this study $(28.2 \%$ for ORIF vs $66.7 \%$ for arthroplasty). This difference is most likely due to the increased age and eligibility of patients undergoing arthroplasty.

Although the data reached conclusions similar to those found in previous research, conflicts still remain. Some studies available found that patients who receive shoulder arthroplasties had reduced complications and revision rates when compared to those who received ORIF [17]. In contrast, Cvetanovich et al. [18] reported that ORIF had the fewest 30-day complications of the three procedures. Manoli et al. [19] also found that patients receiving total shoulder arthroplasty (TSA) were more likely to have higher total hospital charges than patients receiving either hemiarthroplasty or ORIF, and that patients receiving TSA had a shorter length of stay. This directly contrasts with our findings that there was no difference between ORIF and arthroplasty with regards to either hospital charges or length of stay. 
Table 5 Output of binary logistic regression models to identify independent predictors of any complications, extended length of stay and greater hospital charges

\begin{tabular}{llll}
\hline Outcome & Predictor & OR [Cl, 95\%] & $\boldsymbol{p}$ value \\
\hline Any complications & Age & $1.01[1.00-1.02]$ & 0.023 \\
& Gender & $1.04[0.71-1.53]$ & 0.841 \\
& Deyo index & $1.10[0.90-1.34]$ & 0.335 \\
& ORIF vs arthroplasty & $0.67[0.33-1.35]$ & 0.266 \\
Extended length of & Age & $0.99[0.99-1.01]$ & 0.655 \\
stay & Gender & $0.95[0.69-1.31]$ & 0.758 \\
$(>4$ days $)$ & Deyo index & $1.24[1.01-1.51]$ & 0.036 \\
& ORIF vs arthroplasty & $1.01[0.58-1.78]$ & 0.967 \\
Hospital charges & Age & $0.98[0.97-0.99]$ & 0.001 \\
$>\$ 39,387$ & Gender & $0.81[0.53-1.22]$ & 0.303 \\
& Deyo index & $1.31[1.02-1.69]$ & 0.034 \\
& ORIF vs arthroplasty & $1.39[0.68-2.86]$ & 0.366 \\
\hline
\end{tabular}

Statistically significant $p$ values are in italics

According to Bell et al. [20], the rate of surgical treatment for proximal humerus fractures is increasing, but it is currently unknown if equivalent results can be anticipated with open fractures. With an increasing elderly population in the future, it is important to investigate the options for this type of open fracture. This study found no difference in inpatient complication rates between arthroplasty and ORIF for open proximal humerus fractures. To our knowledge, this was the first study to compare these two surgical options for the treatment of these types of fractures.

\section{Limitations}

One limitation of this study is that the Nationwide Inpatient Sample (NIS) only contains information from the patient's hospital stay, and does not contain any followup information or functional outcome of these patients. Additionally, due to the nature of the NIS dataset, we were unable to comment on the classification and types of fractures associated with these surgical interventions, as these data points are not collected within the NIS. Further, the database contains little information regarding the technique used for the ORIF and arthroplasty procedures. Nevertheless, our conclusions are drawn from a large number of patients over 15 years. To our knowledge, our study reports on one of the largest sample sizes of patients with open proximal humerus fractures within the literature, and aligns with views and conclusions from the vast majority of pertinent literature.

\section{Conclusion}

The results from this study help clarify the previous unknowns between two possible procedures, ORIF and arthroplasty, used to treat open fractures of the proximal humerus. While ORIF might be desired for bone preservation in young patients, this study confirms that the risk of in-hospital complications is the same as in those who undergo arthroplasty. The number of comorbidities represented by the Deyo score seems to increase the inpatient complication rate and length of stay. Specifically reporting on open fractures also gives this data a unique perspective not discussed in other studies. Ultimately, further analysis needs to be performed, comparing the long-term complications and clinical outcomes, before further recommendations can be made.

\section{Authors' contributions}

AD carried out study conception, data analysis, study coordination, manuscript drafting, critical revision, and data validation from an orthopaedic surgery standpoint. FSC carried out literature search, data extraction, study coordination, manuscript drafting. CSC carried out data analysis, manuscript revision, study coordination, and data validation from an orthopaedic surgery standpoint. GAB carried out data extraction and analysis, study coordination, manuscript drafting, and literature review. JCM carried out literature review, data analysis, and manuscript drafting. JEM carried out study conception, manuscript revision, data validation from an orthopaedic surgery standpoint. NVS carried out data analysis, manuscript drafting, manuscript revision, proofing/editing, and study coordination. BGD carried out data analysis, manuscript drafting and revision, proofing/editing, study coordination, and data validation from an orthopaedic surgery standpoint. CBP and WPU carried out study conception, manuscript revision, study support, and data validation from an orthopaedic surgery standpoint. All authors were accountable for accuracy and integrity of this manuscript. All authors read and approved the final manuscript.

\section{Competing interests}

Carl B. Paulino is a paid presenter or speaker for DePuy, a Johnson \& Johnson Company. This has not influenced the work presented in this manuscript. All other authors declare that they have no competing interests.

\section{Compliance with ethical standards}

The authors all contributed substantially to the conceptualization, design, execution, and critical review of this study. There are no relationships or conflicts of interest directly related to this paper or that could influence or bias this work. The authors have the following financial relationships to disclose: Dixit — nothing to disclose; Cautela — nothing to disclose; Cooper — nothing to disclose; Beyer — nothing to disclose; Messina — nothing to disclose; Mait — nothing to disclose; Shah — nothing to disclose; Diebo — nothing to disclose; Paulino — DePuy, a Johnson \& Johnson Company — paid speaker or presenter; Urban — nothing to disclose.

\section{Ethics approval and consent to participate}

All procedures performed in studies involving human participants were in accordance with the ethical standards of the institutional and/or national research committee (Institutional Review Board at SUNY Downstate Medical Center) and with the 1964 Helsinki Declaration and its later amendments or comparable ethical standards. As this was a retrospective review, for this type of study formal consent was not required. This study was deemed exempt by our institutional review board due to the de-identified nature of the patient data. This article does not contain any studies with animals performed by any of the authors.

\section{Funding}

This study was performed without any external funding.

IRB approval

SUNY Downstate Medical Center Institutional Review Board. Study \# 661523-1. 


\section{Publisher's Note}

Springer Nature remains neutral with regard to jurisdictional claims in published maps and institutional affiliations.

Received: 1 September 2017 Accepted: 27 November 2017

Published online: 22 August 2018

\section{References}

1. Court-Brown CM, Caesar B (2006) Epidemiology of adult fractures: a review. Injury 37:691-697. https://doi.org/10.1016/j.injury.2006.04.130

2. Kim SH, Szabo RM, Marder RA (2012) Epidemiology of humerus fractures in the United States: nationwide emergency department sample, 2008. Arthritis Care Res 64:407-414. https://doi.org/10.1002/acr.21563

3. Barrett JA, Baron JA, Karagas MR, Beach ML (1999) Fracture risk in the US Medicare population. J Clin Epidemiol 52:243-249

4. Young TB, Wallace WA (1985) Conservative treatment of fractures and fracture-dislocations of the upper end of the humerus. J Bone Joint Surg $\operatorname{Br} 67: 373-377$

5. Guy P, Slobogean GP, McCormack RG (2010) Treatment preferences for displaced three- and four-part proximal humerus fractures. J Orthop Trauma 24:250-254. https://doi.org/10.1097/BOT.0b013e3181 bdc46a

6. Gupta AK, Harris JD, Erickson BJ et al (2015) Surgical management of complex proximal humerus fractures-a systematic review of 92 studies including 4500 patients. J Orthop Trauma 29:54-59. https://doi. org/10.1097/BOT.0000000000000229

7. Misra A, Kapur R, Maffulli N (2001) Complex proximal humeral fractures in adults - a systematic review of management. Injury 32:363-372. https:// doi.org/10.1016/S0020-1383(00)00242-4

8. Bergdahl C, Ekholm C, Wennergren D et al (2016) Epidemiology and patho-anatomical pattern of 2011 humeral fractures: data from the Swedish Fracture Register. BMC Musculoskelet Disord 17:159. https://doi. org/10.1186/s12891-016-1009-8

9. Panagopoulos A, Pantazis K, lliopoulos I et al (2016) Sword-like trauma to the shoulder with open head-splitting fracture of the head. Case Rep Orthop 2016:3539503. https://doi.org/10.1155/2016/3539503

10. Menendez ME, Ring D, Heng M (2015) Proximal humerus fracture with injury to the axillary artery: a population-based study. Injury 46:13671371. https://doi.org/10.1016/j.injury.2015.04.026
11. Thorsness R, lannuzzi J, Noyes K et al (2014) Open reduction and internal fixation versus hemiarthroplasty in the management of proximal humerus fractures. Geriatr Orthop Surg Rehabilit 5:56-62. https://doi. org/10.1177/2151458514527292

12. Rosas S, Law TY, Kurowicki J et al (2016) Trends in surgical management of proximal humeral fractures in the Medicare population: a nationwide study of records from 2009 to 2012. J Shoulder Elb Surg 25:608-613. https://doi.org/10.1016/j.jse.2015.08.011

13. Wild JR, DeMers A, French R et al (2011) Functional outcomes for surgically treated 3- and 4-part proximal humerus fractures. Orthopedics 34:e629-e633. https://doi.org/10.3928/01477447-20110826-14

14. Petrigliano FA, Bezrukov N, Gamradt SC, SooHoo NF (2014) Factors predicting complication and reoperation rates following surgical fixation of proximal humeral fractures. J Bone Joint Surg Am 96:1544-1551. https ://doi.org/10.2106/JBJS.M.01039

15. Neuhaus V, Bot AGJ, Swellengrebel CHJ et al (2014) Treatment choice affects inpatient adverse events and mortality in older aged inpatients with an isolated fracture of the proximal humerus. J Shoulder Elb Surg 23:800-806. https://doi.org/10.1016/j.jse.2013.09.006

16. Zhang AL, Schairer WW, Feeley BT (2014) Hospital readmissions after surgical treatment of proximal humerus fractures: is arthroplasty safer than open reduction internal fixation? Clin Orthop Relat Res 472:2317-2324. https://doi.org/10.1007/s11999-014-3613-y

17. Dai J, Chai Y, Wang C, Wen G (2014) Meta-analysis comparing locking plate fixation with hemiarthroplasty for complex proximal humeral fractures. Eur J Orthop Surg Traumatol 24:305-313. https://doi.org/10.1007/ s00590-013-1179-0

18. Cvetanovich GL, Chalmers PN, Verma NN et al (2016) Open reduction internal fixation has fewer short-term complications than shoulder arthroplasty for proximal humeral fractures. J Shoulder Elb Surg 25(624631):e3. https://doi.org/10.1016/j.jse.2015.09.011

19. Manoli A, Capriccioso CE, Konda SR, Egol KA (2016) Total shoulder arthroplasty for proximal humerus fracture is associated with increased hospital charges despite a shorter length of stay. Orthop Traumatol Surg Res 102:19-24. https://doi.org/10.1016/j.otsr.2015.11.003

20. Bell J-E, Leung BC, Spratt KF et al (2011) Trends and variation in incidence, surgical treatment, and repeat surgery of proximal humeral fractures in the elderly. J Bone Joint Surg Am 93:121-131. https://doi.org/10.2106/ JBJS.I.01505

\section{Submit your manuscript to a SpringerOpen ${ }^{\circ}$ journal and benefit from:}

- Convenient online submission

- Rigorous peer review

- Open access: articles freely available online

- High visibility within the field

- Retaining the copyright to your article

Submit your next manuscript at $\boldsymbol{\nabla}$ springeropen.com 